\title{
Perturbative and nonperturbative quasinormal modes of 4D Einstein-Gauss-Bonnet black holes
}

\author{
Almendra Aragón ${ }^{1, \mathrm{a}}$, Ramón Bécar ${ }^{2, \mathrm{~b}}$, P. A. González ${ }^{1, \mathrm{c}}$, Yerko Vásquez $^{3, \mathrm{~d}}$ \\ ${ }^{1}$ Facultad de Ingeniería y Ciencias, Universidad Diego Portales, Avenida Ejército Libertador 441, Casilla 298-V, Santiago, Chile \\ ${ }^{2}$ Departamento de Ciencias Matemáticas y Físicas, Universidad Católica de Temuco, Temuco, Chile \\ ${ }^{3}$ Departamento de Física y Astronomía, Facultad de Ciencias, Universidad de La Serena, Avenida Cisternas 1200, La Serena, Chile
}

Received: 9 May 2020 / Accepted: 29 July 2020 / Published online: 25 August 2020

(C) The Author(s) 2020

\begin{abstract}
We study the propagation of probe scalar fields in the background of 4D Einstein-Gauss-Bonnet black holes with anti-de Sitter (AdS) asymptotics and calculate the quasinormal modes. Mainly, we show that the quasinormal spectrum consists of two different branches, a branch perturbative in the Gauss-Bonnet coupling constant $\alpha$ and another branch, nonperturbative in $\alpha$. The perturbative branch consists of complex quasinormal frequencies that approximate the quasinormal frequencies of the Schwarzschild AdS black hole in the limit of a null coupling constant. On the other hand, the nonperturbative branch consists of purely imaginary frequencies and is characterized by the growth of the imaginary part when $\alpha$ decreases, diverging in the limit of null coupling constant; therefore they do not exist for the Schwarzschild AdS black hole. Also, we find that the imaginary part of the quasinormal frequencies is always negative for both branches; therefore, the propagation of scalar fields is stable in this background.
\end{abstract}

\section{Contents}

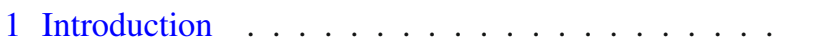

2 Einstein Gauss-Bonnet Black hole in four dimensional AdS spacetime . . . . . . . . . . . 2

3 Scalar field perturbations . . . . . . . . . . . . 3

3.1 Scalar field stability with Dirichlet boundary condition ............. 3

3.2 Numerical analysis . . . . . . . . . . . 4

4 Conclusions ................ . . 7

References . . . . . . . . . . . . . . . 8

a e-mail: almendra.aragon@mail.udp.cl

be-mail: rbecar@uct.cl

c e-mail: pablo.gonzalez@udp.cl

de-mail: yvasquez@userena.cl (corresponding author)

\section{Introduction}

$4 D$ Einstein-Gauss-Bonnet (EGB) gravity has been recently reformulated as the limit $D \rightarrow 4$ of their higher dimensional version when the coupling constant is rescaling as $\alpha \rightarrow \frac{\alpha}{D-4}$ [1]. Thus, the Gauss-Bonnet term shows a nontrivial contribution to the gravitational dynamics. The theory preserves the number of degrees of freedom and remains free from the Ostrogradsky instability. Also, the new theory has stimulated a series of recent research works concerning black holes solutions and the properties of the novel 4D EGB theory; for instance, spherically symmetric black hole solutions were discovered [1], generalizing the Schwarzschild black holes and also being free from a singularity. Additionally, charged black holes in AdS spacetime [2], radiating black holes solutions [3] and an exact charged black hole surrounded by clouds of string was investigated [4]. The generalization of these static black holes to the rotating case was also addressed [5]. On the other hand, regular black holes and the generalization of the BTZ solution in the presence of higher curvature (Gauss-Bonnet and Lovelock) corrections of any order were found in Refs. [6,7], respectively. Also, a $4 D$ Einstein-Lovelock theory was formulated and black hole solutions were studied in $[8,9]$. Interesting physical properties of the black holes in this novel 4D Einstein-GaussBonnet gravity have been investigated such as their thermodynamics [10-14], Hawking radiation and graybody factors $[15,16]$, quasinormal modes and stability [17-21], geodesics motion and shadow [22-24], and electromagnetic radiation from a thin accretion disk from spherically symmetric black holes [25]. However, recent work has raised criticisms about the approach applied in Ref. [1], which arises from the idea of defining a theory from a set of solutions that are obtained by the limit $D \rightarrow 4$ of the $D$-dimensional EGB theory, and there is an active debate on its validity; see for instance [2629]. However, in Refs. [30-32] there have been proposed 
other approaches to obtain a well defined $D \rightarrow 4$ limit of EGB theory, and an action with a set of field equations was found, by using dimensional reduction methods [33,34]. The resulting theory corresponds to a scalar-tensor theory of the Horndeski type. It was shown that all the solutions found in the original paper on $4 D$ EGB theory [1] are also solutions of the new formulation of the theory. In particular, the spherically symmetric Schwarzschild-like solution generated by this theory coincides with the metric of the $D \rightarrow 4$ limit of the $D$-dimensional EGB theory.

In the context of the detection of gravitational waves [35], the quasinormal modes (QNMs) and quasinormal frequencies (QNFs) are important [36-41]. Despite the detected signal being consistent with Einstein gravity [42], there are possibilities for alternative theories of gravity due to the large uncertainties in mass and angular momenta of the ringing black hole [43]. It has been shown that the spectrum of QNMs of theories with higher curvature corrections, such as Einstein-Gauss Bonnet gravity, consists of two different branches [44-48]. One of them has an Einsteinian limit when the Gauss-Bonnet coupling constant $\alpha$ tends to zero, while the other consists of purely imaginary modes of which the damping rate is increasing when $\alpha$ decreases. These modes are qualitatively different from their Einsteinian analogues and they do not exist in the limit $\alpha=0$. Thus, this branch is nonperturbative in $\alpha$ [44]. ${ }^{1}$

The phenomenon of nonperturbative modes seems to be general and independent on the asymptotic behavior of a black hole, of the topology of the event horizon, of the spin of the fields under consideration, and, possibly, even of the particular form of the higher curvature corrections to the General Relativity (GR). Thus, nowadays the study of nonperturbative modes has become a subject of interest, due to the fact that they may lead to a profile qualitatively different from the gravitational ringdown. On the other hand, from the gauge/gravity duality point of view, these modes lead to the eikonal instability of Gauss-Bonnet black holes at some critical values of the coupling constant, so that they determine possible constraints on the holographic applicability of the black hole backgrounds. Moreover, it is worth to mention that the new nonperturbative modes were found for several quite different situations, such as the fourth order in curvature theory [48], asymptotically flat black holes [49-51] and black branes.

In this work we consider 4D Einstein-Gauss-Bonnet black holes with anti-de Sitter (AdS) asymptotics and we study the propagation of scalar fields in such backgrounds, in order to show the existence of nonperturbative QNMs for this kind of theories. We obtain the QNFs numerically by using

\footnotetext{
${ }^{1}$ Calling this branch nonperturbative could sound inappropriate because it is derived by solving the linearized (perturbative) scalar equation.
}

the pseudospectral Chebyshev method [52] which is an effective method to find high overtone modes, and which has been applied for instance in Refs. [53,54]. In spite of the criticisms on the original $4 D \mathrm{EGB}$, it is important to emphasize that the spherically symmetric Schwarzschild-like solution obtained in the $D \rightarrow 4$ limit of the $D$-dimensional EGB theory is also a solution of theories formulated with the well defined limit $D \rightarrow 4$ of EGB theory. Furthermore, it is worth to note that these black holes are also solutions of the semi-classical Einstein equation with Weyl anomaly [55] and for a toy model of Einstein gravity with a Gauss-Bonnet classically "entropic" term mimicking a quantum correction [56]. Therefore, it is worthwhile to perform a study of the physical properties of these black holes, such as the propagation of matter field outside the event horizon. The QNFs of scalar, electromagnetic and gravitational perturbations for this background in asymptotically flat spacetime were obtained recently in Ref. [17], and it was shown that when the coupling constant is positive, the black hole is gravitationally unstable unless the coupling constant is small enough $(0<\alpha \lesssim 0.15)$. The instability develops at high multipole number $\ell$, and therefore is known as an eikonal instability. Also, the negative coupling constant allows for a stable black-hole solution up to relatively large absolute values of $\alpha(0>\alpha \gtrsim-2.0)$. The QNFs of the Dirac field were studied in Ref. [20], and it was shown that the real part of the QNFs is considerably increased, while the damping rate is usually decreasing when the coupling constant increased. Here, besides the perturbative modes, we will find nonperturbative modes in $\alpha$. When $\alpha=0$ the metric corresponds to the Schwarzschild AdS black hole and the QNMs for this geometry were calculated in Ref. [57], where the approach to thermal equilibrium was established, and previously in Ref. [60].

The manuscript is organized as follows: In Sect. 2 we give a brief review of 4D Einstein-Gauss-Bonnet gravity. In Sect. 3 , we study the scalar field stability and calculate numerically the QNFs of scalar field perturbations by using the spectral method. Finally, our conclusions are in Sect. 4.

\section{Einstein Gauss-Bonnet Black hole in four dimensional AdS spacetime}

The Lagrangian of the D-dimensional Einstein-MaxwellGauss-Bonnet theory with the coupling constant re-scaled by $\alpha \rightarrow \frac{\alpha}{D-4}$, is given by the relation [2]

$\mathcal{L}=R-2 \Lambda+\frac{\alpha}{D-4} \mathcal{G}-F^{\mu \nu} F_{\mu \nu}$,

where $\Lambda=-\frac{(D-1)(D-2)}{2 l^{2}}$ is the cosmological constant, $\mathcal{G}=$ $R^{2}-4 R_{\mu \nu} R^{\mu \nu}+R_{\mu \nu \rho \sigma} R^{\mu \nu \rho \sigma}$ is the Gauss-Bonnet term and $F_{\mu \nu}$ is the electromagnetic field tensor. The solutions for a static and spherically symmetric ansatz in an arbitrary 


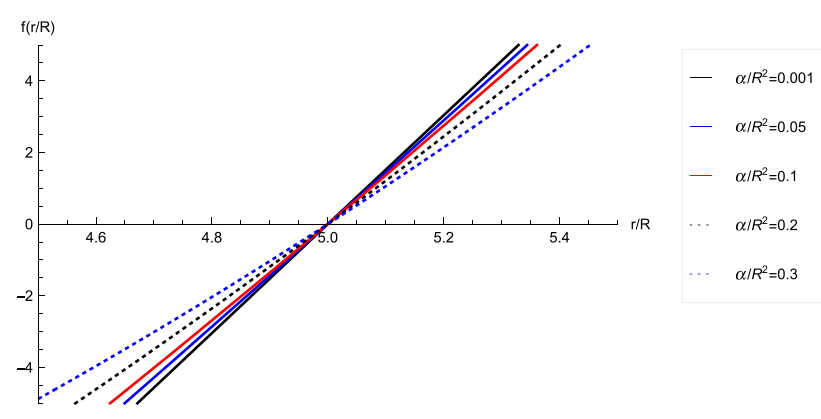

Fig. 1 The behavior of the metric function $f(r / R)$ as a function of $r / R$ for different values of the parameter $\alpha / R^{2}$ with $r_{H} / R=5$, for the region near the event horizon $r_{H} / R$

number of dimensions $D \geq 5$ have the form

$d s^{2}=-f(r) d t^{2}+f(r) d r^{2}+r^{2} d \Omega_{D-2}^{2}$,

where $d \Omega_{D-2}^{2}$ corresponds to a $(D-2)$-dimensional hypersurface. Then, following the prescription given in [1] and taking the limit $D \rightarrow 4$ it is possible to obtain the exact solution representing the 4D Einstein-Maxwell Gauss-Bonnet black hole [2]:

$f(r)=1+\frac{r^{2}}{2 \alpha}\left(1 \pm \sqrt{1+4 \alpha\left(\frac{2 M}{r^{3}}-\frac{Q^{2}}{r^{4}}-\frac{1}{l^{2}}\right)}\right)$,

where $M$ is the mass of black hole and $Q$ is its electric charge. From now on we will consider the uncharged version $Q=0$ of the black hole metric:

$f(r)=1+\frac{r^{2}}{2 \alpha}\left(1 \pm \sqrt{1+4 \alpha\left(\frac{2 M}{r^{3}}-\frac{1}{l^{2}}\right)}\right)$.

Of the two branches of the solution we are interested in the negative branch because physically it is the most interesting one; by taking appropriate limits it is possible to recover some special cases. For instance, when $\alpha \rightarrow 0$, we have the Schwarzschild AdS (SAdS) black hole, and the AdS spacetime $(M=0)$ when $0 \leq \alpha \leq \frac{l^{2}}{4}$, and for null cosmological constant we have the seminal result found in [1] with the coupling parameter $\alpha>0$. It is worth to mention that similar metrics were found previously in the context of quantum corrections to gravity $[55,56,61]$.

The black hole horizon $r_{H}$ corresponds to the largest root of $f(r)=0$. In Fig. 1 we show the behavior of $f(r)$ of different values of $\alpha / R^{2}$.

It is convenient to measure all the quantities in the units of the same dimension, so we express $M$ as a function of the event horizon $r_{H}$ :

$M=-\frac{\Lambda r_{H}^{3}}{6}+\frac{r_{H}}{2}+\frac{\alpha}{2 r_{H}}$,

where the cosmological constant $\Lambda=-\frac{3}{l^{2}}$ can be expressed in terms of the AdS radius $R$, which is defined by $f(r \rightarrow$

$$
\begin{aligned}
& \infty)=r^{2} / R^{2}, \text { as } \\
& \Lambda=-\frac{3\left(R^{2}-\alpha\right)}{R^{4}} .
\end{aligned}
$$

\section{Scalar field perturbations}

The QNMs of scalar perturbations in the background of the metric (4) are given by the scalar field solution of the KleinGordon equation,

$\frac{1}{\sqrt{-g}} \partial_{\mu}\left(\sqrt{-g} g^{\mu \nu} \partial_{\nu} \varphi\right)=m^{2} \varphi$,

with suitable boundary conditions for a black hole geometry. In the above expression $m$ is the mass of the scalar field $\varphi$. Now, by means of the ansatz

$\varphi=e^{-i \omega t} R(r) Y(\Omega)$,

the Klein-Gordon equation reduces to

$$
\begin{aligned}
& f(r) R^{\prime \prime}(r)+\left(f^{\prime}(r)+2 \frac{f(r)}{r}\right) R^{\prime}(r) \\
& \quad+\left(\frac{\omega^{2}}{f(r)}-\frac{\ell(\ell+1)}{r^{2}}-m^{2}\right) R(r)=0,
\end{aligned}
$$

where $\ell=0,1,2, \ldots$ represents the azimuthal quantum number and the prime denotes the derivative with respect to $r$. Now, defining $R(r)=\frac{F(r)}{r}$ and by using the tortoise coordinate $r^{*}$ defined by $d r^{*}=\frac{d r}{f(r)}$, the Klein-Gordon equation can be written as a one-dimensional Schrödinger equation,

$\frac{d^{2} F\left(r^{*}\right)}{d r^{* 2}}-V_{e f f}(r) F\left(r^{*}\right)=-\omega^{2} F\left(r^{*}\right)$,

with an effective potential $V_{\text {eff }}(r)$, which is parametrically thought of as $V_{e f f}\left(r^{*}\right)$, given by

$V_{e f f}(r)=f(r)\left(\frac{f^{\prime}(r)}{r}+\frac{\ell(\ell+1)}{r^{2}}+m^{2}\right)$.

The effective potential diverges at spatial infinity and it is positive definite everywhere outside the event horizon; see Fig. 2. Therefore, we will consider as a boundary condition that the scalar field vanishes at the asymptotic region (the Dirichlet boundary condition).

3.1 Scalar field stability with Dirichlet boundary condition

In order to address the stability of the propagation of scalar fields, we follow a general argument given in Ref. [57]. So, by defining

$\psi(r)=e^{i \omega r^{*}} F(r)$, 


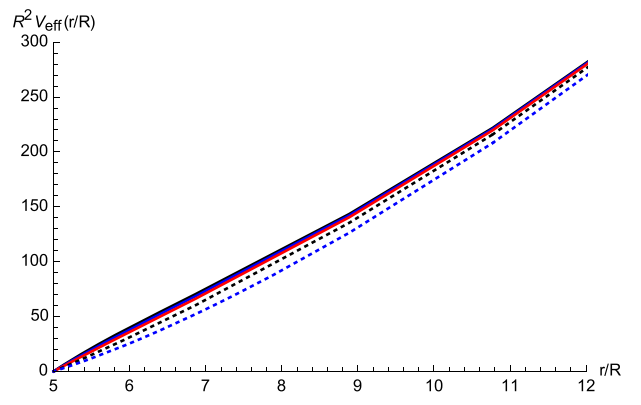

$-\alpha / R^{2}=0.001$

$-\alpha / R^{2}=0.05$

$-\alpha / R^{2}=0.1$

$\cdots \quad \alpha / R^{2}=0.2$

$\cdots \quad \alpha / R^{2}=0.3$

Fig. 2 The behavior of the effective potential $R^{2} V_{\text {eff }}$ as a function of $r / R$ for different values of the parameter $\alpha / R^{2}$ with $r_{H} / R=5, \ell=0$, and $m R=0.1$

and inserting this expression in the Schrödinger-like equation (10) yields

$\frac{d}{d r}\left(f(r) \frac{d \psi(r)}{d r}\right)-2 i \omega \frac{d \psi(r)}{d r}-\frac{V_{e f f}(r)}{f(r)} \psi(r)=0$.

Then, multiplying Eq. (13) by $\psi^{*}$ and performing integration by parts, and using the Dirichlet boundary condition for the scalar field at spatial infinity, one can obtain the expression

$$
\begin{gathered}
\int_{r_{+}}^{\infty} d r\left(f(r)\left|\frac{d \psi}{d r}\right|^{2}+\frac{V_{e f f}(r)}{f(r)}|\psi|^{2}\right) \\
=-\frac{|\omega|^{2}\left|\psi\left(r=r_{H}\right)\right|^{2}}{\operatorname{Im}(\omega)} .
\end{gathered}
$$

In general, the QNFs are complex, where the real part represents the frequency of the oscillation and the imaginary part describes the rate at which this oscillation is damped, with the stability of the scalar field being guaranteed if the imaginary part is negative. The potential (5) is positive outside the horizon and then the left hand side of (14) is strictly positive, which demand that $\operatorname{Im}(\omega)<0$, and then we conclude that the stability of the propagation of a scalar field respecting Dirichlet boundary conditions is stable.

\subsection{Numerical analysis}

In this section we will solve numerically the differential equation (9) in order to compute the QNFs for the black hole described by the metric by using the pseudospectral Chebyshev method; see for instance [52]. First, under the change of variable $y=1-r_{H} / r$ the radial Eq. (9) becomes

$$
\begin{aligned}
& (1-y)^{4} f(y) R^{\prime \prime}(y)+(1-y)^{4} f^{\prime}(y) R^{\prime}(y) \\
& \quad+\left(\frac{\omega^{2} r_{H}^{2}}{f(y)}-\ell(\ell+1)(1-y)^{2}-m^{2} r_{H}^{2}\right) R(y)=0,
\end{aligned}
$$

where the prime denotes derivative with respect to $y$. In the new coordinate the event horizon is located at $y=0$ and the spatial infinity at $y=1$. Now, we consider the boundary
Table 1 Some lowest quasinormal frequencies $\omega R$ for the branches nonperturbative and perturbative in $\alpha$, in the background of the black hole with $r_{H} / R=5, \ell=0$ and $m=0$

\begin{tabular}{lll}
\hline$\alpha / R^{2}$ & Nonperturbative QNFs & Perturbative QNFs \\
\hline 0.01 & $-108.89879 i$ & $9.34676-13.50717 i$ \\
0.03 & $-51.24661 i$ & $9.07679-13.90768 i$ \\
0.06 & $-31.31146 i$ & $8.61373-14.63611 i$ \\
0.15 & $-14.99874 i$ & $7.95765-18.24388 i$ \\
\hline
\end{tabular}

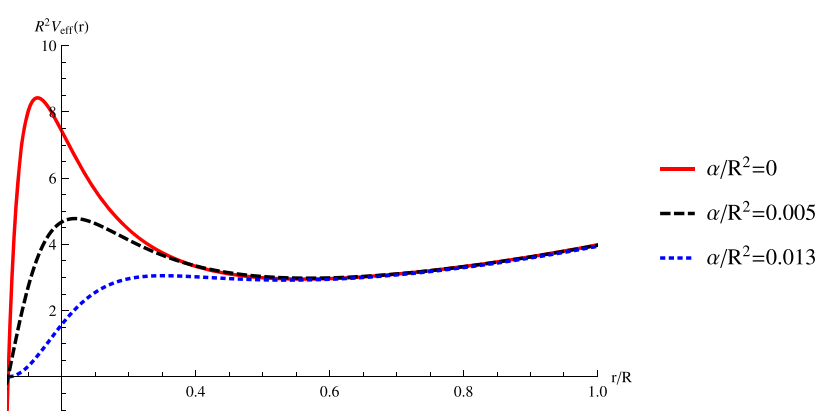

Fig. 3 The effective potential for small black holes for $r_{H} / R=0.12$, $m R=0, \ell=0$, and different values of $\alpha / R$

conditions. In the neighborhood of the horizon $(\mathrm{y} \rightarrow 0)$ the function $R(y)$ behaves as

$R(y)=C_{1} e^{-\frac{i \omega r_{H}}{f^{\prime}(0)} \ln y}+C_{2} e^{\frac{i \omega r_{H}}{f^{\prime}(0)} \ln y}$.

Here, the first term represents an ingoing wave and the second term represents an outgoing wave near the black hole horizon. Imposing the requirement of having only ingoing waves on the horizon, we fix $C_{2}=0$. On the other hand, at infinity the function $R(y)$ behaves as

$$
\begin{aligned}
R(y)= & D_{1}(1-y)^{\frac{3}{2}+\sqrt{\left(\frac{3}{2}\right)^{2}+m^{2} R^{2}}} \\
& +D_{2}(1-y)^{\frac{3}{2}-\sqrt{\left(\frac{3}{2}\right)^{2}+m^{2} R^{2}}} .
\end{aligned}
$$

So, imposing the requirement that the scalar field vanishes at infinity requires $D_{2}=0$. Taking into account the above behaviors of the scalar field at the horizon and at spatial infinity we define

$R(y)=e^{-\frac{i \omega r_{H}}{f^{\prime}(0)} \ln y}(1-y)^{\frac{3}{2}+\sqrt{\left(\frac{3}{2}\right)^{2}+m^{2} R^{2}}} F(y)$.

Then, by inserting this last expression in Eq. (15) we obtain an equation for the function $F(y)$, which we solve numerically employing the pseudospectral Chebyshev method. The solution for the function $F(y)$ is assumed to be a finite linear combination of the Chebyshev polynomials, and it is inserted in the differential equation for $F(y)$. The interval $[0,1]$ is discretized at the Chebyshev collocation points. Then the 


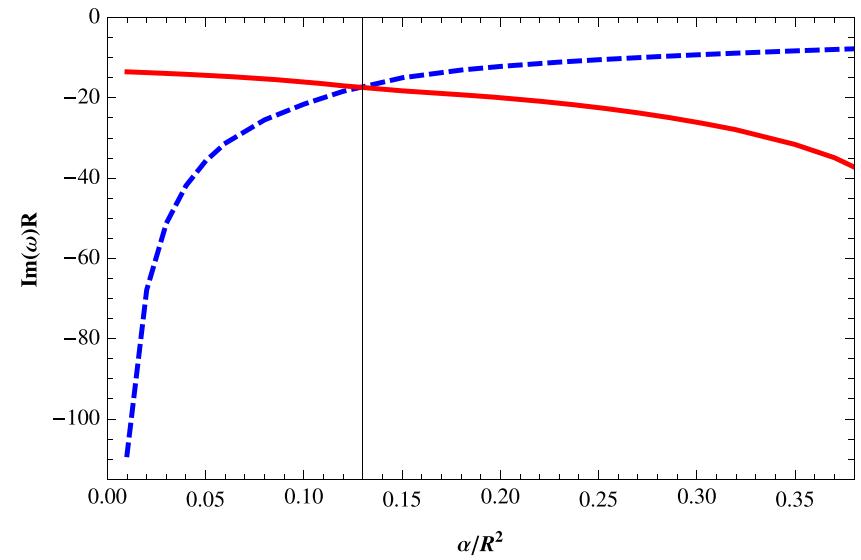

Fig. 4 Behavior of the imaginary part of the QNFs for the nonperturbative in $\alpha$ modes (blue dashed line) and perturbative modes (red continuous line) of a massless scalar field (left panel). The vertical line

differential equation is evaluated at each collocation point. Thus, a system of algebraic equations is obtained which corresponds to a generalized eigenvalue problem and it is solved numerically for $\omega$.

In Fig. 4, left panel, we show the behavior of the imaginary part of the QNFs for a massless scalar field with $\ell=0$ as a function of $\alpha / R^{2}$, for the ratio $r_{H} / R=5$, and also we show the real part of the QNFs, under the same considerations, right panel. We can observe the existence of two branches. One of them corresponds to the branch perturbative in $\alpha$ (red continuous line), which consists of complex QNFs that in the limit $\alpha \rightarrow 0$ approximate the QNFs of a massless scalar field in the background of the SAdS black hole [57]. On the other hand, the branch nonperturbative in $\alpha$ (blue dashed line) consists of purely imaginary QNFs, which diverge in the limit $\alpha \rightarrow 0$; therefore they do not exist for SAdS black hole. We show in Table 1 some numerical values of the QNFs. Also, we observe that the imaginary part of the QNFs is always negative for both branches; therefore, the propagation of massless scalar fields is stable in this background. It is worth to mention that there is a critical value $\alpha=\alpha_{c}$, where the curves intersect and the two branches have the same imaginary part, for $\alpha$ lower than the critical value the nonperturbative branch decays faster than the perturbative branch, while that for $\alpha$ greater than the critical value, the behavior is opposite, i.e., the pertubative branch decays faster than the nonperturbative branch; thus the nonperturbative branch dominates in this case. The real part of the perturbative QNFs, see Fig. 4, shows a smooth behavior and we observe that the frequency of the oscillation decreases when $\alpha / R^{2}$ increases. In addition, we observe that there is a small range where the frequency increases slightly and then decreases again.

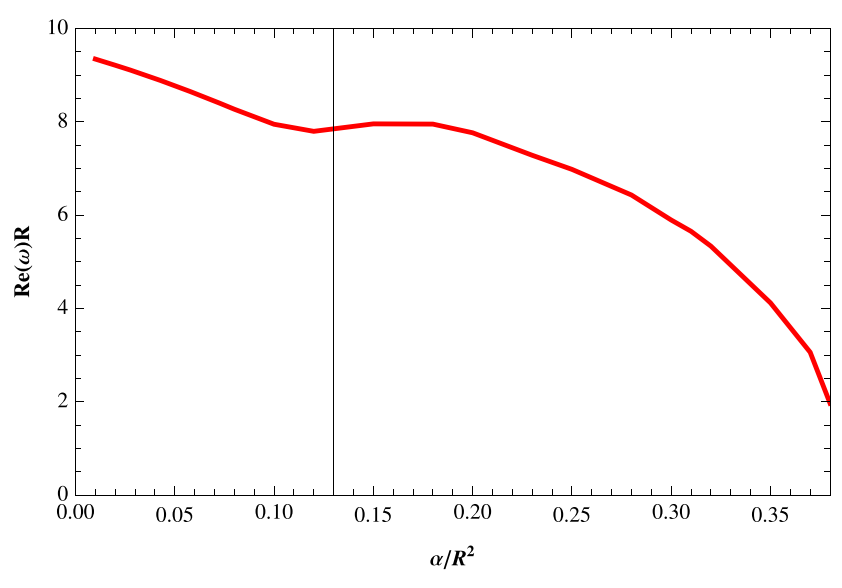

corresponds to the critical value of $\alpha$ where the curves cross. Behavior of the real part of QNFs for the perturbative modes of a massless scalar field, with $\ell=0$ as a function of $\alpha / R^{2}$, for $r_{H} / R=5$ (right panel)

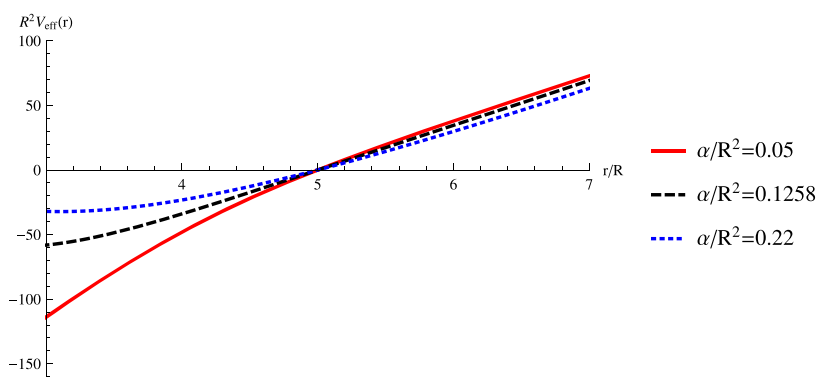

Fig. 5 The effective potential for $r_{H} / R=5, m R=0, \ell=0$ and $\alpha / R^{2}=0.05<\alpha_{c} / R^{2}, \alpha / R^{2}=\alpha_{c} / R^{2}=0.1258$ and $\alpha / R^{2}=$ $0.22>\alpha_{c} / R^{2}$

We observe that for $\ell=m=0$ there exist two different potentials ${ }^{2}$. There is one that looks like a potential barrier near the outside horizon that is well increasing, see Fig. 3, while the other is a monotonically increasing function like Fig. 2. The former shows a small SAdS black hole is featuring, whereas the latter indicates a large SAdS black hole. In [58] was showed that a potential-step type provides the purely imaginary QNFs, while the potential-barrier type gives the complex QNFs of a scalar field for the charged dilaton black hole. The presence of the bump near the horizon explains clearly why the QNFs for gravitational and electromagnetic perturbations of the small SAdS black hole are complex in [59]. For the 4D Einstein-Gauss-Bonnet AdS black hole, we observe a similar behavior of the potential; for small black holes and small values of $\alpha$ we note the presence of a potential barrier, which disappears when $r_{H} / R$ or $\alpha$ increases; see Fig. 3. Thus, it is possible to explain the two kinds of QNFs of a scalar field around the 4D Einstein-Gauss-Bonnet

${ }^{2}$ We thank the referee for pointing out this behavior of the potential to us. 

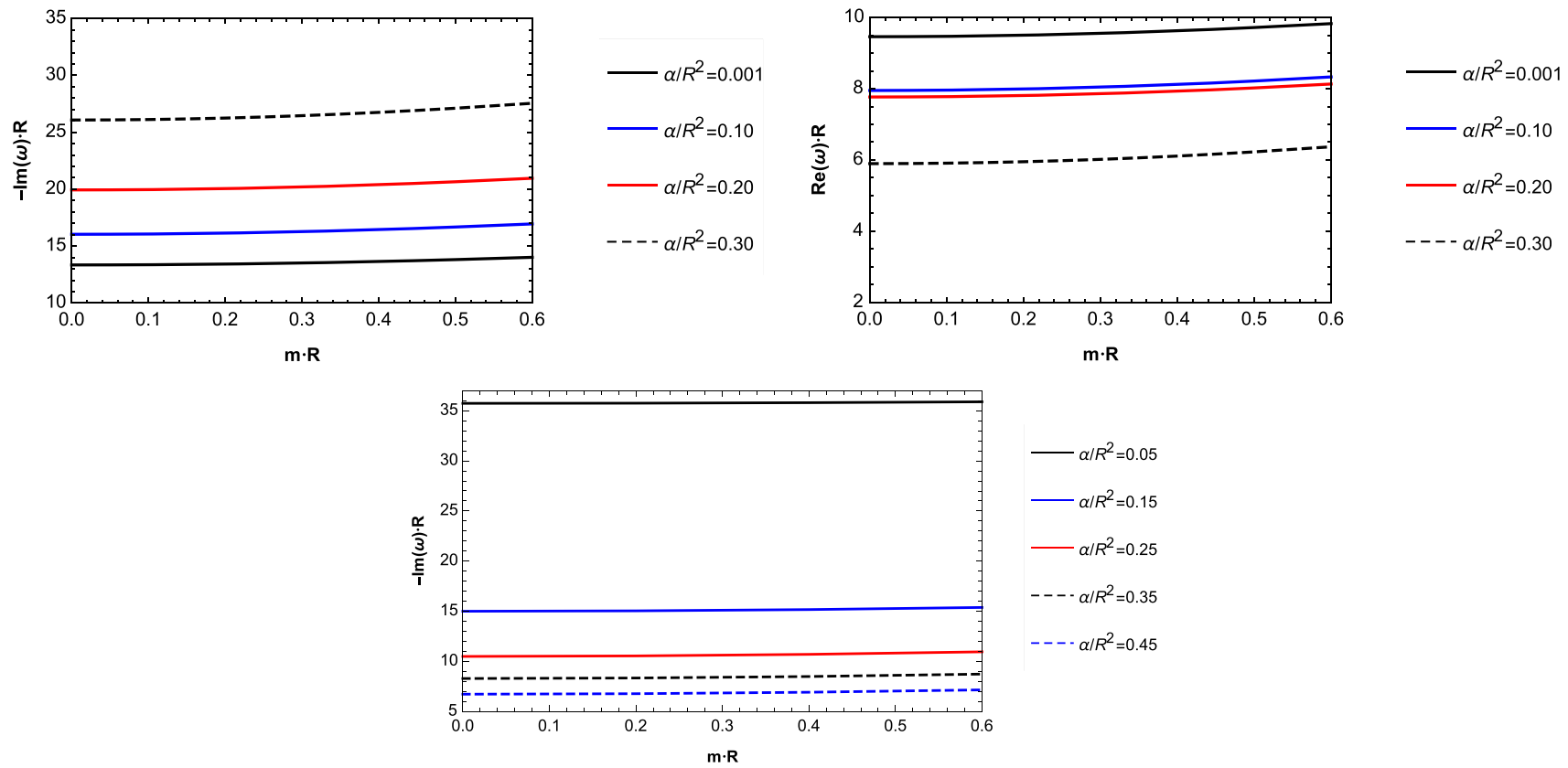

Fig. 6 Behavior of the QNFs as a function of $m R$, of a massive scalar field with $\ell=0$ for different values of $\alpha / R^{2}$ and $r_{H} / R=5$. Modes perturbative in $\alpha$ (top panel), left plot for the imaginary part and right plot for the real part; and nonperturbative modes (bottom panel)
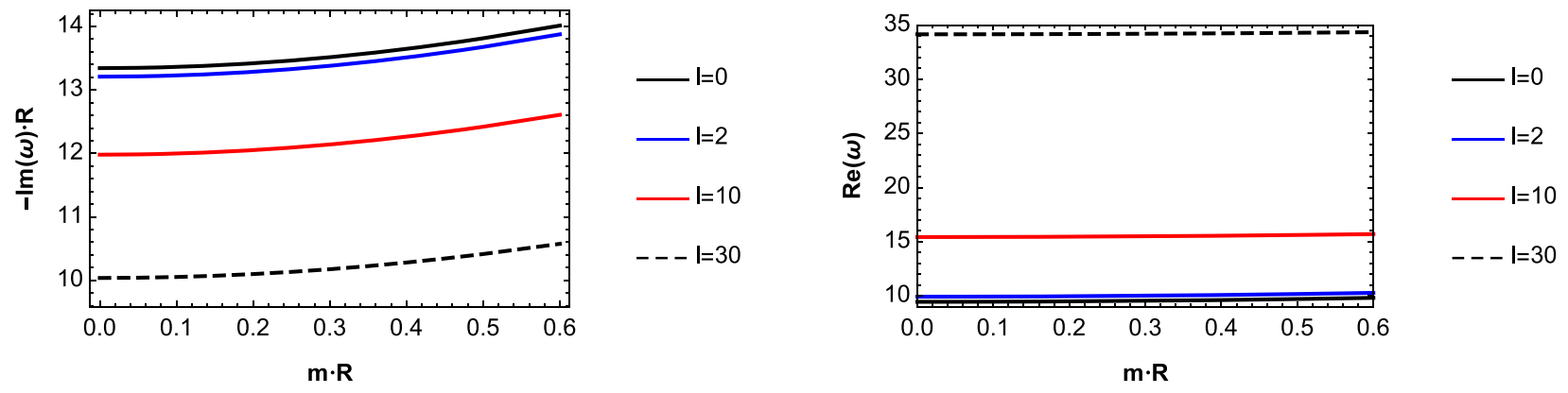

Fig. 7 Behavior of modes perturbative in $\alpha$ as a function of $m R$, with $r_{H} / R=5, \alpha / R^{2}=0.001$ and for different values of the angular number $\ell=0,2,10,30$. The left plot is for the imaginary part and the right plot for the real part of the quasinormal spectrum

AdS black holes by identifying their potentials. While the potential-barrier type gives the complex QNFs, the monotonically increasing type gives the purely imaginary QNFs. On the other hand, as mentioned before, in Fig. 4 we observe that for $\alpha<\alpha_{c}$ the complex QNFs dominate, while for $\alpha>\alpha_{c}$ the purely imaginary QNFs dominate. Interestingly, we found that this behavior is related to the change of concavity of the potential at the event horizon. We found that for $\alpha=0$ the second derivative of the effective potential evaluated at the horizon is always negative, and it is given by

$$
\begin{aligned}
& V_{e f f}^{\prime \prime}\left(r_{H}\right) \\
& =-\frac{\left.6(5+2 \ell(\ell+1))\left(r_{H} / R\right)^{2}+18\left(r_{H} / R\right)^{4}+2\left(4+3 \ell(\ell+1)+m^{2} R^{2}\left(r_{H} / R\right)^{2}\right)\right)}{r_{H}^{4}} .
\end{aligned}
$$

However, for $\alpha \neq 0$, the concavity of the potential at the event horizon can be positive, and we note that the potential has a point of inflection, see Fig. 5, at the event horizon for $\alpha=\alpha_{c}$ where the curves in Fig. 4 intersect. For $\alpha<\alpha_{c}$ the potential has a negative concavity at the event horizon, such as for the SAdS black hole, and the complex QNF dominates, while for $\alpha>\alpha_{c}$ the concavity of the potential at the event horizon is positive and the purely imaginary QNF dominates. The change of sign of $V_{e f f}^{\prime \prime}\left(r_{H}\right)$ when $\alpha$ increases is attributed to the effect of the higher order curvature terms on the metric.

Now, in order to analyze the behavior of the QNFs of massive scalar field, we plot in Fig. 6 their behavior for the lowest angular number $\ell=0$ as a function of $m R$, and for different values of $\alpha / R^{2}$. We can observe the complex branch (top panel) and the purely imaginary branch (bottom panel), which belong to the perturbative and nonperturbative branches, respectively. For the perturbative branch, we can observe that there is a faster decay rate of the perturbations when the mass of the scalar field increases, and the frequency 

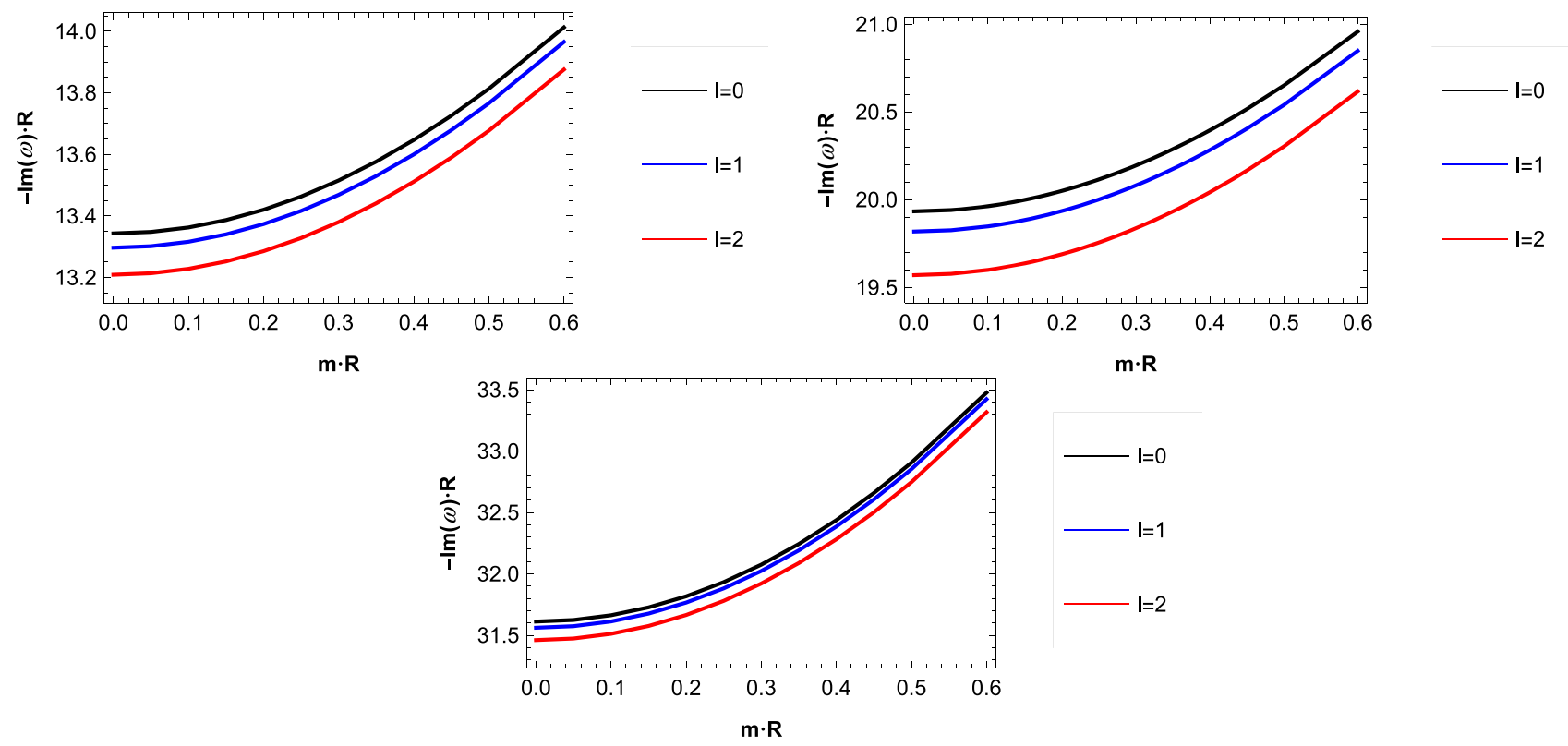

Fig. 8 Behavior of imaginary part of the perturbative in $\alpha$ modes as a function of $m R$, with $r_{H} / R=5$, for low values of angular number $\ell=0,1,2$. Left top panel for $\alpha / R^{2}=0.001$, right top panel for $\alpha / R^{2}=0.20$ and bottom panel for $\alpha / R^{2}=0.35$

of the oscillations increases too. Also, the decay rate and the frequency of the oscillation increases when $\alpha / R^{2}$ decreases, for a fixed value of $m R$. On the other hand, for the nonperturbative branch the decay rate increases slightly when the scalar field mass increases. Also, there is a faster decay when $\alpha / R^{2}$ decreases.

Now, in order to analyze the behavior of the QNFs of massive scalar field, we plot in Fig. 7 their behavior, for low angular numbers $\ell=0,2$ and high angular numbers $\ell=10,30$ as a function of $m R$ with $\alpha / R^{2}=0.001$ fixed. For the perturbative branch, we can observe that there is a lower decay rate and the frequency of the oscillations increases when the angular number $\ell$ increases. In Fig. 8, we observe that the behavior is similar for low angular numbers $\ell=$ $0,1,2$ and different values of $\alpha / R^{2}$.

\section{Conclusions}

In this work, we considered 4D Einstein-Gauss-Bonnet black holes in AdS spacetime as backgrounds and we studied the propagation of probe scalar fields. We found numerically the quasinormal frequencies for different values of the Gauss-Bonnet coupling constant $\alpha / R^{2}$, the multipole number $\ell$ and the mass of the scalar field $m R$ by using the pseudospectral Chebyshev method. Mainly, we found two branches of QNFs, a branch perturbative in the coupling constant $\alpha$, and another branch, nonperturbative in $\alpha$, that is, they do not exist in the limit $\alpha=0$.
The branch nonperturbative in $\alpha$ is characterized by purely imaginary QNFs with a faster decay when $\alpha / R^{2}$ decreases, while for the branch perturbative in $\alpha$ the QNFs tend to the QNFs of the Schwarzschild AdS black hole when $\alpha \rightarrow 0$; a lower decay is observed when $\alpha / R^{2}$ decreases. Also, we found that the imaginary part of the QNFs is always negative for the two branches; therefore, the propagation of scalar fields is stable for the asymptotically AdS 4D EinsteinGauss-Bonnet black hole. There are two different behaviors of the potential; one looks like a potential barrier near the outside horizon-well-increasing, while the other is a monotonically increasing function. The former shows a small SAdS black hole featuring, whereas the latter indicates a large SAdS black hole. For small black holes and small values of $\alpha$ we note the presence of a potential barrier, which disappears when $r_{H} / R$ or $\alpha$ increases. Therefore, it is possible to explain the two kinds of QNFs of a scalar field around the 4D Einstein-Gauss-Bonnet AdS black holes by identifying their potentials, i.e., while the potential-barrier type gives the complex QNFs, the monotonically increasing type gives the purely imaginary QNFs.

Interestingly, we found that there is a critical value of $\alpha=\alpha_{c}$, where the two branches have the same imaginary part, and for values of $\alpha$ lower than the critical value the nonperturbative branch decays faster than the perturbative branch. Meanwhile for values of $\alpha / R^{2}$ greater than the critical value, the behavior is opposite, i.e., the perturbative branch decays faster than the nonperturbative branch; thus the nonperturbative branch dominates in this case. Additionally, we have found that for $\alpha=0$ the second derivative of 
the effective potential evaluated at the horizon is always negative, while for $\alpha \neq 0$, the concavity of the potential at the event horizon can be positive, and we note that the potential has a point of inflection at the event horizon for $\alpha=\alpha_{c}$. For $\alpha<\alpha_{c}$ the potential has negative concavity at the event horizon, such as for the SAdS black hole, and the complex QNF dominates, while for $\alpha>\alpha_{c}$ the concavity of the potential at the event horizon is positive and the purely imaginary QNF dominates. The change of sign of the second derivative of the effective potential evaluated at the horizon when $\alpha$ increases is attributed to the effect of the higher order curvature terms on the metric.

We showed that the phenomenon of nonperturbative modes arises for scalar field perturbations for the $4 \mathrm{D}$ Einstein-Gauss-Bonnet theory, by extending the presence of nonperturbative modes to the other theory. On the other hand, the inverse of the imaginary part of the fundamental quasinormal frequency is related, through the AdS/CFT duality, to the thermalization time of the quantum states in the boundary field theory [57]. In addition, it was found in $[45,48]$ that black holes with AdS asymptotics in theories with higher curvature terms can help to describe the intermediate 't Hooft coupling in the dual field theory; thus, we hope that the results obtained in this work can have applications along this line.

Acknowledgements We would like to thank the referee for his/her careful review of the manuscript and his/her valuable comments and suggestions which helped us to improve the manuscript considerably. Y.V. acknowledges support by the Dirección de Investigación y Desarrollo de la Universidad de La Serena, Grant No. PR18142.

Data Availability Statement This manuscript has no associated data or the data will not be deposited. [Authors' comment:This is a theoretical paper without associated data.]

Open Access This article is licensed under a Creative Commons Attribution 4.0 International License, which permits use, sharing, adaptation, distribution and reproduction in any medium or format, as long as you give appropriate credit to the original author(s) and the source, provide a link to the Creative Commons licence, and indicate if changes were made. The images or other third party material in this article are included in the article's Creative Commons licence, unless indicated otherwise in a credit line to the material. If material is not included in the article's Creative Commons licence and your intended use is not permitted by statutory regulation or exceeds the permitted use, you will need to obtain permission directly from the copyright holder. To view a copy of this licence, visit http://creativecomm ons.org/licenses/by/4.0/.

Funded by SCOAP ${ }^{3}$.

\section{References}

1. D. Glavan, C. Lin, Phys. Rev. Lett. 124(8), 081301 (2020). arXiv:1905.03601 [gr-qc]

2. P.G.S. Fernandes, arXiv:2003.05491 [gr-qc]

3. S.G. Ghosh, R. Kumar, arXiv:2003.12291 [gr-qc]
4. D.V. Singh, S.G. Ghosh, S.D. Maharaj, arXiv:2003.14136 [gr-qc]

5. S.W. Wei, Y.X. Liu, arXiv:2003.07769 [gr-qc]

6. A. Kumar, R. Kumar, arXiv:2003.13104 [gr-qc]

7. R.A. Konoplya, A. Zhidenko, arXiv:2003.12171 [gr-qc]

8. R.A. Konoplya, A. Zhidenko, Phys. Rev. D 101(8), 084038 (2020). arXiv:2003.07788 [gr-qc]

9. R.A. Konoplya, A. Zhidenko, arXiv:2005.02225 [gr-qc]

10. K. Hegde, A. Naveena Kumara, C.L. A. Rizwan, A.K.M., M. S. Ali, arXiv:2003.08778 [gr-qc]

11. S.A. Hosseini Mansoori, arXiv:2003.13382 [gr-qc]

12. S.W. Wei, Y.X. Liu, arXiv:2003.14275 [gr-qc]

13. D.V. Singh, S. Siwach, arXiv:2003.11754 [gr-qc]

14. B. Eslam Panah, K. Jafarzade, arXiv:2004.04058 [hep-th]

15. C.Y. Zhang, P.C. Li, M. Guo, arXiv:2003.13068 [hep-th]

16. R.A. Konoplya, A.F. Zinhailo, arXiv:2004.02248 [gr-qc]

17. R.A. Konoplya, A.F. Zinhailo, arXiv:2003.01188 [gr-qc]

18. R.A. Konoplya, A. Zhidenko, arXiv:2003.12492 [gr-qc]

19. A.K. Mishra, arXiv:2004.01243 [gr-qc]

20. M.S. Churilova, arXiv:2004.00513 [gr-qc]

21. C.Y. Zhang, S.J. Zhang, P.C. Li, M. Guo, arXiv:2004.03141 [gr-qc]

22. M. Guo, P.C. Li, arXiv:2003.02523 [gr-qc]

23. M. Heydari-Fard, M. Heydari-Fard, H. Sepangi, arXiv:2004.02140 [gr-qc]

24. R. Roy, S. Chakrabarti, arXiv:2003.14107 [gr-qc]

25. C. Liu, T. Zhu, Q. Wu, arXiv:2004.01662 [gr-qc]

26. M. Gurses, T.C. Sisman, B. Tekin, arXiv:2004.03390 [gr-qc]

27. S. Mahapatra, arXiv:2004.09214 [gr-qc]

28. F.W. Shu, arXiv:2004.09339 [gr-qc]

29. S.X. Tian, Z.H. Zhu, arXiv:2004.09954 [gr-qc]

30. H. Lu, Y. Pang, arXiv:2003.11552 [gr-qc]

31. P.G.S. Fernandes, P. Carrilho, T. Clifton, D.J. Mulryne, arXiv:2004.08362 [gr-qc]

32. R.A. Hennigar, D. Kubiznak, R.B. Mann, C. Pollack, arXiv:2004.09472 [gr-qc]

33. R.B. Mann, S.F. Ross, Class. Quant. Gravit. 10, 1405 (1993). arXiv:9208004 [gr-qc]

34. T. Kobayashi, arXiv:2003.12771 [gr-qc]

35. B.P. Abbott et al. [LIGO Scientific and Virgo Collaborations], Phys. Rev. Lett. 116(6), 061102 (2016)

36. T. Regge, J.A. Wheeler, Phys. Rev. 108, 1063 (1957)

37. F.J. Zerilli, Phys. Rev. D 2, 2141 (1970)

38. K.D. Kokkotas, B.G. Schmidt, Living Rev. Rel. 2, 2 (1999). arXiv:9909058 [gr-qc]

39. H.P. Nollert, Class. Quant. Gravit. 16, R159 (1999)

40. R.A. Konoplya, A. Zhidenko, Rev. Mod. Phys. 83, 793 (2011). arXiv: 1102.4014 [gr-qc]

41. E. Berti, V. Cardoso, A.O. Starinets, Class. Quant. Gravit. 26, 163001 (2009). arXiv:0905.2975 [gr-qc]

42. B.P. Abbott et al. [LIGO Scientific and Virgo Collaborations], Phys. Rev. Lett. 116(22), 221101 (2016)

43. R. Konoplya, A. Zhidenko, Phys. Lett. B 756, 350 (2016)

44. P.A. Gonzalez, R.A. Konoplya, Y. Vasquez, Phys. Rev. D 95(12), 124012 (2017). arXiv:1703.06215 [gr-qc]

45. S. Grozdanov, N. Kaplis, A.O. Starinets, JHEP 1607, 151 (2016). arXiv: 1605.02173 [hep-th]

46. R.A. Konoplya, A. Zhidenko, Phys. Rev. D 95(10), 104005 (2017). arXiv: 1701.01652 [hep-th]

47. P.A. Gonzalez, Y. Vasquez, R.N. Villalobos, Phys. Rev. D 98(6), 064030 (2018). arXiv: 1807.11827 [gr-qc]

48. S. Grozdanov, A.O. Starinets, arXiv:1611.07053 [hep-th]

49. T. Takahashi, J. Soda, Prog. Theor. Phys. 124, 911 (2010). arXiv: 1008.1385 [gr-qc]

50. G. Dotti, R.J. Gleiser, Phys. Rev. D 72, 044018 (2005). arXiv:0503117 [gr-qc]

51. R.J. Gleiser, G. Dotti, Phys. Rev. D 72, 124002 (2005). arXiv:0510069 [gr-qc] 
52. J.P. Boyd, Chebyshev and Fourier Spectral Methods. Dover Books on Mathematics, 2nd edn. Dover Publications, Mineola, NY (2001)

53. S.I. Finazzo, R. Rougemont, M. Zaniboni, R. Critelli, J. Noronha, JHEP 1701, 137 (2017). arXiv:1610.01519 [hep-th]

54. P.A. González, E. Papantonopoulos, J. Saavedra, Y. Vásquez, Phys. Rev. D 95(6), 064046 (2017). arXiv:1702.00439 [gr-qc]

55. R.G. Cai, L.M. Cao, N. Ohta, JHEP 1004, 082 (2010). arXiv:0911.4379 [hep-th]

56. G. Cognola, R. Myrzakulov, L. Sebastiani, S. Zerbini, Phys. Rev. D 88(2), 024006 (2013). arXiv:1304.1878 [gr-qc]
57. G.T. Horowitz, V.E. Hubeny, Phys. Rev. D 62, 024027 (2000). arXiv:9909056 [hep-th]

58. Y.S. Myung, Y.W. Kim, Y.J. Park, Eur. Phys. J. C 58, 617 (2008). arXiv:0809.1933 [gr-qc]

59. V. Cardoso, J.P.S. Lemos, Phys. Rev. D 64, 084017 (2001). arXiv:gr-qc/0105103

60. J. Chan, R.B. Mann, Phys. Rev. D 55, 7546-7562 (1997). arXiv:gr-qc/9612026 [gr-qc]

61. Y. Tomozawa, arXiv:1107.1424 [gr-qc] 